\title{
Strong Coupling Solution to Schrödinger Equation: The Mixing of States
}

\author{
L. M. Garrido and L. Navarro \\ Instituto de Fisica Teórica and G.I.F.T., Barcelona, Spain
}

(Received 6 October 1969)

\begin{abstract}
In this paper we give some ideas that can be useful to solve Schrödinger equations in the case when the Hamiltonian contains a large term. We obtain an expansion of the solution in reciprocal powers of the large coupling constant. The procedure followed consists in considering that the small part of the Hamiltonian engenders a motion adiabatic to the motion generated by the large part of the same.
\end{abstract}

\section{INTRODUCTION}

We define strong coupling, stating that the perturbation expansion is not valid. Then we pretend to obtain the solutions to the equations of motion in negative powers of the coupling constant $g$. Such an expansion has not been achieved so far and constitutes a crucial problem of elementary-particles dynamics. It is assumed nowadays that perturbation expansion is not valid; however, most of the calculation performed at the present time to discover the symmetry of elementary particles assume the validity of perturbation theory, an assumption that probably is not true.

We cannot state without discrimination that in the case of strong coupling we have to expand the solutions of the equation of motion into negative powers of the coupling constant. The coupling constant, being large, may be multiplied in the solution by factors that are small, which yields a small product. Thus, for strong coupling the general solution has to be expanded into negative powers of the coupling constant when multiplied by small factors. Most likely there is an intermediate region in which the solution has to be evaluated by variational methods, as it corresponds to intermediate effective coupling.

As is well known, it is hoped that, for the strong coupling approximation, the eigenstates of the large part of the Hamiltonian play the most important role. What we pretend in this paper is to find out, in powers of the reciprocal of the coupling constant, the mixing that the small part of the Hamiltonian produces among the eigenstates of the large part. The procedure followed consists in considering that the small part of the Hamiltonian engenders a motion adiabatic ${ }^{1}$ to the motion generated by the large part of the same. This paper is no more than an introduction to the abovementioned problem.

Indeed, we do not solve the problem completely, since we do not obtain completely a series of negative powers of the coupling constant; but this paper is a first approximation to the desired solution.
The Schrödinger equation which is studied is

$$
i \hbar \frac{\partial}{\partial t}|t\rangle=\left(H_{0}+g H_{1}\right)|t\rangle,
$$

where $H_{0}$ and $H_{1}$ are time independent, while the coupling constant $g$ is large.

The solution to this equation is exactly

$$
|t\rangle=\exp \left[\frac{t}{i \hbar}\left(H_{0}+g H_{1}\right)\right]|0\rangle,
$$

but, written in this way, we do not see how the eigenstates of $H_{1}$ are mixed amongst themselves. In what follows, we pretend to solve such a problem.

\section{STRONG COUPLING SOLUTIONS TO SCHRÖDINGER EQUATION}

Essentially, we want to solve the equation

$$
i \hbar \frac{\partial}{\partial t} U(t)=\left(H_{0}+g H_{1}\right) U(t)
$$

for the unitary operator $U(t)$, since the time evolution of the state vector $|t\rangle$ at instant $t$ is given by

$$
|t\rangle=U(t)|0\rangle
$$

when we suppose that $t=0$ is the time origin. Correspondingly, we impose on $U(t)$ the following boundary condition:

$$
U(0)=I .
$$

The total Hamiltonian $H=H_{0}+g H_{1}$ contains the dimensionless coupling constant $g$, which we suppose to be large. This implies that $g H_{1}$ gives a large contribution to the time derivative of the evolution operator $U(t)$, i.e., such an operator generates a fast time dependence of $U(t)$. Perturbation expansion in powers of $g$ is not valid, since $g$ is large.

We assume also, for the sake of concreteness, that $H_{0}$ and $H_{1}$ do not have an explicit time dependence. The study of the cases when $H_{0}$ and $H_{1}$ may have explicit time dependence yields much more complicated solutions and no better insight of the method used in this paper is gained. Besides, we should remember 
that for most physical applications $H_{0}$ and $H_{1}$ do not depend on time explicitly, though they may have a dynamical time dependence; this is the case of quantum field theory.

Since the contribution of $g H_{1}$ to $U(t)$ is very large, it is sensible to suppose that the eigenvectors of $H_{1}$ play an important role in the expansion of $U(t)$ in powers of $g^{-1}$. This evident fact means that we will have difficulties in interpreting the physical meaning of the solution found, since,usually, free or undressed particles are the eigenvalues of the Hamiltonian $H_{0}$, which in this case has a much smaller contribution to the motion than $g H_{1}$.

Therefore, we use the eigenvectors of $H_{1}$ as the basis for the representation of the solution. So we assume that the following equation has been solved,

$$
H_{1}|E\rangle=E|E\rangle,
$$

and that the eigenvectors $|E\rangle$ form a complete set in the sense that the identity operator is given by

$$
I=\sum_{E}|E\rangle\langle E| .
$$

Let $A$ be any time-dependent operator. We can split it into a diagonal part $A^{D}$ and a nondiagonal part $A^{N}$, in relation to the basis of our representation, the eigenvectors $|E\rangle$. So we have

$$
A=A^{D}+A^{N}
$$

where

$$
\begin{aligned}
& A^{D} \equiv \sum_{E}|E\rangle\langle E|A| E\rangle\langle E|, \\
& A^{N} \equiv \sum_{E, E^{\prime}}\left\{|E\rangle\left\langle E|A| E^{\prime}\right\rangle\left\langle E^{\prime}\right|\left(-\delta_{E^{\prime}, E^{\prime}}+1\right)\right\} .
\end{aligned}
$$

It is very easy to check that diagonal parts of any two operators commute, while the corresponding nondiagonal parts generally do not commute; the product of any two diagonal parts of operators is a diagonal operator, while the product of two nondiagonal parts of operators may have diagonal and nondiagonal parts. We also have from the definitions above

$$
\left\langle E\left|A^{D}\right| E\right\rangle=\langle E|A| E\rangle .
$$

The diagonal part of the Hamiltonian $H$ is

$$
H^{D}=H_{0}^{D}+g H_{1},
$$

and its nondiagonal part is

$$
H^{N}=H_{0}^{N} .
$$

We solve the time equation for $U(t)$ exactly for $H^{D}$ and treat $H^{N}$ as the perturbation that mixes the eigenvectors of $H_{1}$, as we have already done in preceding papers. ${ }^{2-4}$ Accordingly, we write

$$
U(t)=S_{D}(t) S(t),
$$

where $S_{D}(t)$ is a diagonal operator in our chosen representation, i.e., an exact solution of the following equation,

$$
i \hbar \frac{\partial}{\partial t} S_{D}(t)=\left(H_{0}^{D}+g H_{1}\right) S_{D}(t),
$$

and $S(t)$ is an operator that contains diagonal and nondiagonal parts in general and that we have to expand in negative powers of $g$.

We have to impose the conditions $S_{D}(0)=I$ and $S(0)=I$. We have

$$
S_{D}(t)=\exp \left\{\frac{t}{i \hbar}\left(H_{0}^{D}+g H_{1}\right)\right\},
$$

the exact solution of the equation for $S_{D}(t)$. The equation for $S(t)$ is

$$
i \hbar \frac{\partial}{\partial t} S(t)=H_{0}^{N}[t] S(t)
$$

when, as usually occurs, the effective-perturbation Hamiltonian is defined by

$$
H_{0}^{N}[t]=S_{D}^{-1}(t) H_{0}^{N} S_{D}(t)
$$

which is well known since $S_{D}(t)$ has been obtained exactly and $H_{0}^{N}$ can be evaluated. Then

$$
\begin{aligned}
S(t)=I & +\frac{1}{i \hbar} \int_{0}^{t} d t^{\prime} H_{0}^{N}\left[t^{\prime}\right] \\
& +\frac{1}{(i \hbar)^{2}} \int_{0}^{t} d t^{\prime} H_{0}^{N}\left[t^{\prime}\right] \int_{0}^{t^{\prime}} d t^{\prime \prime} H_{0}^{N}\left[t^{\prime \prime}\right]+\cdots,
\end{aligned}
$$

as is well known. We have to show that, indeed, the terms of the expansion (3) as a series in powers of $H_{0}^{N}$ contain negative powers of $g$ only, when $g$ is large.

We define

$$
\begin{aligned}
\bar{A}(t) \equiv & \frac{1}{i \hbar} \int_{0}^{t} d t^{\prime} S_{D}^{-1}\left(t^{\prime}\right) A\left(t^{\prime}\right) S_{D}\left(t^{\prime}\right) \\
= & \frac{1}{i \hbar} \int_{0}^{t} d t^{\prime} S_{D}^{-1}\left(t^{\prime}\right) A^{N}\left(t^{\prime}\right) S_{D}\left(t^{\prime}\right) \\
& +\frac{1}{i \hbar} \int_{0}^{t} d t^{\prime} S_{D}^{-1}\left(t^{\prime}\right) A^{D}\left(t^{\prime}\right) S_{D}\left(t^{\prime}\right) \\
= & S_{D}^{-1}(t)_{\delta}\left[A^{N}\right] S_{D}(t)+\left[A^{D}\right]_{\delta},
\end{aligned}
$$

where

$$
\begin{aligned}
{ }_{\delta}\left[A^{N}\right] \equiv S_{D}(t) & \left\{\frac { 1 } { i \hbar } \sum _ { E , E ^ { \prime } } \int _ { 0 } ^ { t } d t ^ { \prime } \left(\operatorname { e x p } \frac { t } { i \hbar } \left[\left\langle E\left|H_{0}^{D}+g H_{1}\right| E\right\rangle\right.\right.\right. \\
- & \left.\left.\left\langle E^{\prime}\left|H_{0}^{D}+g H_{1}\right| E^{\prime}\right\rangle\right]\right) \\
& \left.\times|E\rangle\left\langle E\left|A^{N}\left(t^{\prime}\right)\right| E^{\prime}\right\rangle\left\langle E^{\prime}\right\}\right\} S_{D}^{-1}(t)
\end{aligned}
$$

and

$$
\left[A^{D}\right]_{\delta}=\frac{1}{i \hbar} \int_{0}^{t} d t^{\prime} A^{D}\left(t^{\prime}\right)
$$


Integrating (4) by parts, we obtain

$$
\begin{aligned}
{ }_{\delta}\left[A^{N}\right]= & \sum_{E, E^{\prime}}\left(\left\langle E\left|H_{0}^{D}+g H_{1}\right| E\right\rangle\right. \\
& \left.-\left\langle E^{\prime}\left|H_{0}^{D}+g H_{1}\right| E^{\prime}\right\rangle\right)^{-1} \\
& \times\left\{S _ { D } ( t ) \left[\left(\operatorname { e x p } \frac { t } { i \hbar } \left[\left\langle E\left|H_{0}^{D}+g H_{1}\right| E\right\rangle\right.\right.\right.\right. \\
& \left.\left.-\left\langle E^{\prime}\left|H_{0}^{D}+g H_{1}\right| E^{\prime}\right\rangle\right]\right) \\
& \left.\times|E\rangle\left\langle E\left|A^{N}(t)\right| E^{\prime}\right\rangle\left\langle E^{\prime}\right|\right]_{0}^{t} S_{D}^{-1}(t) \\
& -S_{D}(t)\left[\int _ { 0 } ^ { t } d t ^ { \prime } \left(\operatorname { e x p } \frac { t ^ { \prime } } { i \hbar } \left[\left\langle E\left|H_{0}^{D}+g H_{1}\right| E\right\rangle\right.\right.\right. \\
& \left.\left.-\left\langle E^{\prime}\left|H_{0}^{D}+g H_{1}\right| E^{\prime}\right\rangle\right]\right) \\
& \left.\left.\times|E\rangle\left\langle E\left|\frac{d}{d t^{\prime}} A^{N}\left(t^{\prime}\right)\right| E^{\prime}\right\rangle\left\langle E^{\prime}\right|\right] S_{D}^{-1}(t)\right\} \\
= & O(1 / g),
\end{aligned}
$$

because we suppose, as usual, that $A(t)$ and its derivatives are bounded operators.

Now we can evaluate every term in (3):

$$
\begin{aligned}
I_{1} & =\frac{1}{i \hbar} \int_{0}^{t} d t^{\prime} H_{0}^{N}\left[t^{\prime}\right]=\frac{1}{i \hbar} \int_{0}^{t} d t^{\prime} S_{D}^{-1}\left(t^{\prime}\right) H_{0}^{N} S_{D}\left(t^{\prime}\right) \\
& =S_{D}^{-1}(t)_{\delta}\left[H_{0}^{N}\right] S_{D}(t)=O(1 / g), \\
I_{2} & =\frac{1}{(i \hbar)^{2}} \int_{0}^{t} d t^{\prime} H_{0}^{N}\left[t^{\prime}\right] \int_{0}^{t^{\prime}} d t^{\prime \prime} H_{0}^{N}\left[t^{\prime \prime}\right] \\
& =\frac{1}{i \hbar} \int_{0}^{t} d t^{\prime} S_{D}^{-1}\left(t^{\prime}\right) H_{0}^{N}\left[H_{0}^{N}\right] S_{D}\left(t^{\prime}\right) \\
& =S_{D}^{-1}(t)_{\delta}\left[H_{0}^{N}\left[H_{0}^{N}\right]\right] S_{D}(t)+\frac{1}{i \hbar} \int_{0}^{t} d t^{\prime}\left[H_{0 \delta}^{N}\left[H_{0}^{N}\right]\right]^{D} .
\end{aligned}
$$

The first term in the expression of $I_{2}$ is of the order of $g^{-2}$ and the second is of the order of $g^{-3}$ because

$$
\tau / h \simeq 1 / g^{2}
$$

by definition of the coupling constant $g$, where $\tau$ is approximately the time during which the interaction takes place. Similarly,

$$
\begin{aligned}
I_{3}= & \frac{1}{i \hbar} \int_{0}^{t} H_{0}^{N}\left[t^{\prime}\right] I_{2}\left(t^{\prime}\right) \\
= & S_{D}^{-1}(t)_{\delta}\left[H_{0 \delta}^{N}\left[H_{0 \delta}^{N}\left[H_{0}^{N}\right]\right]\right] S_{D}(t) \\
& +\frac{1}{i \hbar} \int_{0}^{t} d t^{\prime}\left[H_{0 \delta}^{N}\left[H_{0 \delta}^{N}\left[H_{0}^{N}\right]\right]\right]^{D} .
\end{aligned}
$$

Therefore, the degree of approximation of every term can be simply obtained by adding to the number of $\delta$ twice the number of integrals. The terms in $I_{3}$ are

$$
O\left(1 / g^{3}\right), \quad O\left(1 / g^{4}\right) .
$$

Therefore, the unitary evolution operator in the second-order approximation, for instance, is

$$
U(t)=\left\{I+{ }_{\delta}\left[H_{0}^{N}\right]+{ }_{\delta}\left[H_{0 \delta}^{N}\left[H_{0}^{N}\right]\right]+O\left(1 / g^{3}\right)\right\} S_{D}(t) .
$$

Evidently, it is quite easy to continue with the calculation of further terms in the expansion of $S(t)$. We see that successive terms contain higher and higher negative powers of the dimensionless coupling constant $g$.

A very interesting case is that in which both $H_{0}$ and $H_{1}$ can be simultaneously diagonalized. Then our procedure results are considerably simplified (the same occurs in the ordinary perturbation theory). The expression for $U(t)$ can be reduced to

$$
U(t)=S_{D}(t) .
$$

Then, in this special case, it is not possible to obtain the evolution operator as an expansion in reciprocal powers of the large coupling constant using this procedure. But when this occurs, the problem is easier than usual and frequently can be solved, as in Example $I$ in the next section.

We should remark that it is essential for the validity of this expansion that the difference

$$
\left\langle E^{\prime}\left|H_{0}^{D}+g H_{1}\right| E^{\prime}\right\rangle-\left\langle E\left|H_{0}^{D}+g H_{1}\right| E\right\rangle
$$

be large for any two values of $E$ and $E^{\prime}$. If it were small, the method would not be acceptable, since the denominators in ${ }_{\delta}[A]$ would not be large.

The time dependence of $U(t)$ is very fast, since the exponent of $S_{D}(t)$ contains a constant without dimensions which is large. But $S(t)$ will not necessarily vary rapidly with time. We have here two time scales: the fast one generated by $H^{D}$ and the slow one generated by $H^{N}$. We separate the two time dependences, fast and slow, to solve the problem considering the slow time dependence as producing a small perturbation compared to the fast time variation. Both motions are adiabatic to each other. The method is similar to those used by us in preceding papers to treat adiabatic motions.

\section{EXAMPLES}

\section{A. Example I}

Consider two identical linear oscillators with spring constant $k$ and an interaction potential given by $g x_{1} x_{2}$, where $x_{1}$ and $x_{2}$ are the oscillator variables, and $g$ is large. The total Hamiltonian is

where

$$
\mathscr{H}=\mathscr{H}_{0}+g \mathscr{H}_{1},
$$

$$
\mathcal{H}_{0}=-\frac{\hbar^{2}}{2 m}\left(\frac{\partial^{2}}{\partial x_{1}^{2}}+\frac{\partial^{2}}{\partial x_{2}^{2}}\right)+\frac{k}{2}\left(x_{1}^{2}+x_{2}^{2}\right)
$$


and

$$
\mathfrak{H}_{1}=x_{1} x_{2} .
$$

The energy levels of $\mathcal{H}_{0}$ are thus given by

where

$$
E_{0}=\left(n_{1}+\frac{1}{2}\right) \hbar \omega+\left(n_{2}+\frac{1}{2}\right) \hbar \omega,
$$

$$
\omega^{2}=k / m .
$$

As we really have a two-dimensional problem, it is advantageous to use a $\mathcal{N} \otimes \mathcal{N}$ representation, $\mathcal{N}$ being the occupation number of each oscillator. Then the unperturbated eigenfunctions are

$$
\left|n_{1} n_{2}\right\rangle=n_{1}|1.0\rangle+n_{2}|0.1\rangle \text {. }
$$
is

The Hamiltonian matrix of the unperturbed motion

$$
\mathscr{H}_{0}=\frac{3}{2} \hbar \omega\left(\begin{array}{ll}
1 & 0 \\
0 & 1
\end{array}\right)
$$

and the Hamiltonian matrix of the perturbation, taken with respect to the unperturbed states, is

where

$$
\mathscr{H}_{1}=\left(\begin{array}{ll}
0 & a \\
a & 0
\end{array}\right)
$$

$$
a=\left\langle 1.0\left|H_{1}\right| 0.1\right\rangle=g\left\langle 0\left|x_{1}\right| 1\right\rangle\left\langle 1\left|x_{2}\right| 0\right\rangle=\hbar / 2 m \omega,
$$

as may be deduced from the quantum-mechanical version of the virial theorem.

In order to apply the approximation given by (6), it is necessary to make a transformation of state vector bases to achieve a new representation in which $\mathfrak{H}_{1}$ is diagonal. This matrix transformation is

$$
A=\frac{1}{i \sqrt{2}}\left(\begin{array}{rr}
1 & 1 \\
1 & -1
\end{array}\right)
$$

Then

$$
\begin{aligned}
& H_{0}=A^{-1} \mathcal{H}_{0} A=\frac{3}{2} \hbar \omega\left(\begin{array}{ll}
1 & 0 \\
0 & 1
\end{array}\right), \\
& H_{1}=A^{-1} \mathcal{H}_{1} A=\left(\begin{array}{cc}
a & 0 \\
0 & -a
\end{array}\right),
\end{aligned}
$$

and

$$
{ }_{\delta}\left[H_{0}\right]=S_{D}(t)\left[\int_{0}^{t} d t^{\prime} S_{D}^{-1}\left(t^{\prime}\right) H_{0}^{N} S_{D}\left(t^{\prime}\right)\right] S_{D}^{-1}(t)=0 .
$$

Formula (5) gives

$$
U(t)=S_{D}(t)=\exp \left[-\frac{i}{\hbar} t\left(H_{0}+g H_{1}\right)\right],
$$

or, in the original representation,

$$
U(t)=\exp \left[-\frac{i}{\hbar} t\left(\mathfrak{H}_{0}+\mathfrak{g H}_{1}\right)\right]
$$

In this case we have not achieved an expansion in powers of $g^{-1}$. But, owing to the simultaneous diagonalization of $\mathscr{H}_{0}$ and $\mathscr{H}_{1}$, the problem can be easily solved. Defining new variables $u$ and $v$ by

$$
x_{1}=(u+v) / 2, \quad x_{2}=(u-v) / 2,
$$

we express the Hamiltonian as

$H=-\frac{\hbar^{2}}{2 m}\left(\frac{\partial^{2}}{\partial u^{2}}+\frac{\partial^{2}}{\partial v^{2}}\right)+\frac{1}{2}(k+g) u^{2}+\frac{1}{2}(k-g) v^{2}$.

The exact energy levels are thus given by

$$
E=n_{1} \hbar \omega_{1}+n_{2} \hbar \omega_{2}+(\hbar / 2)\left(\omega_{1}+\omega_{2}\right),
$$

where $n_{1}$ and $n_{2}$ are positive integers and

$$
\omega_{1}^{2}=(k-g) / m, \quad \omega_{2}^{2}=(k+g) / m,
$$

and the eigenfunctions are

$$
\left|n_{1} n_{2}\right\rangle=F_{n_{1}}(u) F_{n_{2}}(v),
$$

where the $F$ 's are simple harmonic oscillator wavefunctions.

Actually, this problem has nothing to do with the procedure that we introduced in Sec. 2. It is easy enough to be solved with a simple change of the variables.

We have included it for two reasons: First, it serves to introduce the notation that we are going to employ with some further complications in the next example, which is the one that demonstrates the validity of our method. Secondly, it represents the special case in which $\mathcal{H}_{0}$ and $\mathscr{H}_{1}$ can be diagonalized simultaneously. In this case, our procedure is not available, but then we have shown that the problem is easy to solve, as we anticipated in Sec. 2.

\section{B. Example II}

In the foregoing example, it has not been possible to check the validity of the approximation suggested by formula (6). In order to verify it, we consider two different oscillators strongly coupled. In this case,

$$
\begin{aligned}
& \mathscr{H}_{0}=\frac{3}{2} \hbar\left(\begin{array}{cc}
\omega_{1} & 0 \\
0 & \omega_{2}
\end{array}\right), \\
& \mathscr{H}_{1}=\left(\begin{array}{ll}
0 & a \\
a & 0
\end{array}\right),
\end{aligned}
$$

where

$$
a=\left\langle 1.0\left|\mathfrak{H}_{1}\right| 0.1\right\rangle=\hbar(2 m)^{-1}\left(\omega_{1} \omega_{2}\right)^{-\frac{1}{2}} .
$$

The diagonalization of $\mathfrak{H}_{1}$ as in the preceding example can be done by transforming both Hamiltonians with the matrix

$$
A=\frac{1}{i \sqrt{2}}\left(\begin{array}{rr}
1 & 1 \\
1 & -1
\end{array}\right)
$$


The new Hamiltonians are

$$
\begin{aligned}
& H_{0}=A^{-1} \mathfrak{H}_{0} A=\frac{3}{4} \hbar\left(\begin{array}{cc}
\omega_{1}+\omega_{2} & \omega_{2}-\omega_{1} \\
\omega_{2}-\omega_{1} & \omega_{1}+\omega_{2}
\end{array}\right), \\
& H_{1}=A^{-1} \mathfrak{H}_{1} A=\left(\begin{array}{cc}
a & 0 \\
0 & -a
\end{array}\right)=\hbar(2 m)^{-1}\left(\omega_{1} \omega_{2}\right)^{-\frac{1}{2}}\left(\begin{array}{cc}
1 & 0 \\
0 & -1
\end{array}\right), \\
& H=\frac{\hbar}{2}\left(\begin{array}{cc}
\frac{3}{2}\left(\omega_{1}+\omega_{2}\right)+g(m)^{-1}\left(\omega_{1} \omega_{2}\right)^{-\frac{1}{2}} & \frac{3}{2}\left(\omega_{2}-\omega_{1}\right) \\
\frac{3}{2}\left(\omega_{2}-\omega_{1}\right) & \frac{3}{2}\left(\omega_{1}+\omega_{2}\right)-g(m)^{-1}\left(\omega_{1} \omega_{2}\right)^{-\frac{1}{2}}
\end{array}\right) .
\end{aligned}
$$

The first-order approximation of formula (6) is given by

where

$$
U(t)=\left(1+{ }_{\delta}\left[H_{0}^{N}\right]\right) S_{D}(t)
$$

and

$$
S_{D}(t)=\exp (t / i \hbar)\left(H_{0}^{D}+g H_{1}\right)
$$

$$
H_{0}^{D}=\frac{3}{4} \hbar\left(\begin{array}{cc}
\omega_{1}+\omega_{2} & 0 \\
0 & \omega_{1}+\omega_{2}
\end{array}\right)
$$

The validity of the method can be checked by evaluating $H_{0}^{N}$ and verifying that it is of the order of $g^{-1}$.

Now we may easily calculate

Integration yields

$$
S_{D}^{-1}(t) H_{0}^{N} S_{D}(t)=\frac{3}{4} \hbar\left(\omega_{2}-\omega_{1}\right)\left(\begin{array}{cc}
0 & \exp \left[-g t(i m)^{-1}\left(\omega_{1} \omega_{2}\right)^{-\frac{1}{2}}\right]
\end{array}\right) .
$$

$$
\begin{aligned}
{ }_{\delta}\left[H_{0}^{N}\right] & =S_{D}(t)\left[\int_{0}^{t} d t^{\prime} S_{D}^{-1}\left(t^{\prime}\right) H_{0}^{N} S_{D}\left(t^{\prime}\right)\right] S_{D}^{-1}(t) \\
& =\frac{3}{4} \hbar\left(\omega_{2}-\omega_{1}\right) \frac{i m \sqrt{\omega_{1} \omega_{2}}}{g}\left(\begin{array}{cc}
0 & 1-\exp \left[g t(i m)^{-1}\left(\omega_{1} \omega_{2}\right)^{-\frac{1}{2}}\right] \\
1-\exp \left[-g t(i m)^{-1}\left(\omega_{1} \omega_{2}\right)^{-\frac{1}{2}}\right] & 0
\end{array}\right),
\end{aligned}
$$

in accordance with (5).

The above expression is of the order of $1 / g$ because $\omega_{2} \neq \omega_{1}$. Thus,

$$
U(t)=\left\{\left(\begin{array}{ll}
1 & 0 \\
0 & 1
\end{array}\right)+\frac{3}{4} \hbar\left(\omega_{2}-\omega_{1}\right) \frac{i m \sqrt{\omega_{1} \omega_{2}}}{g}\left(\begin{array}{cc}
0 & 1-\exp \left[g t(i m)^{-1}\left(\omega_{1} \omega_{2}\right)^{-\frac{1}{2}}\right] \\
1-\exp \left[-g t(i m)^{-1}\left(\omega_{1} \omega_{2}\right)^{-\frac{1}{2}}\right] & 0
\end{array}\right) S_{D} .\right.
$$

Expression (7) allows us to solve this problem in the first order and prove our assertion about the nondiagonal character of ${ }_{\delta}\left[H_{0}^{N}\right]$.

\section{CONCLUSION}

We have found how the eigenvalues of $H_{1}$ mix. We have found an expression of $S(t)$ that, expanded in powers of $g^{-1}$, is the term that indicates how the eigenvalues of $H_{1}$ mix.

The process developed in this paper will be applied in a future paper to evaluate the mass renormalization of a nucleon coupled to a cloud of mesons when the coupling constant is large.

1 A. Lenard, Ann. Phys. 6, 261 (1959).

${ }^{2}$ L. M. Garrido, J. Math. Phys. 5, 335 (1964).

${ }^{3}$ L. Navarro and L. M. Garrido, Proc. Phys. Soc., Ser. 2,1, 326 (1968).

${ }^{4}$ L. Navarro and L. M. Garrido, Physica 39, 361 (1968). 